\title{
DESARROLLO DE LA COMPETENCIA BÁSICA “COMUNICACIÓN LINGÜÍSTICA" A TRAVÉS DEL CUENTO MOTOR Y EL CUADERNO DEL ALUMNO EN LA ASIGNATURA DE EDUCACIÓN FÍSICA
}

\author{
Eva Rivas Martínez \\ C.E.I.P. "Miguel Ángel Sáinz" (La Rioja) \\ Javier Terroba Valdemoros \\ I.E.S. "Celso Díaz" (La Rioja)
}

\begin{abstract}
RESUMEN: Con este artículo pretendemos aportar información sobre las competencias básicas, elemento nuevo que se ha introducido en el currículo, derivado del desarrollo de la Ley Orgánica de Educación (LOE, 2006) en el sistema educativo español, concretamente sobre la que hace referencia a la comunicación lingüística.

Por otra parte, este componente curricular se origina como consecuencia de los estudios realizados tanto por la Unión Europea como por la OCDE, en un intento de aportar referencias comunes para los sistemas educativos europeos.

Con el fin de no limitarnos a lo meramente teórico, se ha aportado una experiencia didáctica de Educación Física -a partir de ahora, EF-, en la que puede comprobarse el desarrollo de la competencia básica de comunicación lingüística a través del cuaderno del alumno y la lectura de un cuento motor, en una Unidad Didáctica destinada al desarrollo de contenidos pertenecientes al Bloque de Contenidos 3: Actividades físicas artístico-expresivas, titulada "Explosión de expresiones", destinada al 2º nivel de educación primaria.
\end{abstract}

ABSTRACT: In this article we provide information on the treatment of key competences from the area of Physical Education, a new element that has been introduced into the curriculum involved in the development of the Organic Law of Education (LOE) in the Spanish educational system, particularly on the referring to linguistic communication.

Moreover, this curricular component arises as a result of studies conducted by the European Union and by the Organization for Cooperation and Economic Development in an attempt to provide a common reference for European education systems. 
To avoid falling into the merely theoretical, it has provided a learning experience in physical education, where can be checked the development of key competences of linguistic communication, through motor storytelling and the student activity book, in a Didactic Unit for the development of the contents belonging to the 3rd block of contents: Physical Activities artistic and expressive, entitled "Expression Explosion", intended for the 2nd level of Primary Education.

PALABRAS CLAVE: Competencias básicas, comunicación lingüística, DeSeCo, cuaderno del alumno, cuento motor, expresión corporal, LOE, enseñanzas mínimas.

KEYWORDS: Key competences, linguistic communication, DeSeCo, notebook, motor story, activities artistic and expressive, LOE, minimun teaching requirements.

\section{INTRODUCCIÓN}

La nueva normativa educativa española, en consonancia con las directrices surgidas de la Unión Europea, introduce como un elemento más del currículo el concepto de competencia básica. Este concepto novedoso hay que integrarlo dentro de nuestras programaciones didácticas, y esto es algo que la mayor parte del profesorado desconoce. Es por ello que con este artículo se pretenda, por un lado, dar unos conocimientos elementales sobre las competencias básicas, y por otro, incluir, a modo de ejemplo, una propuesta didáctica para el desarrollo de la competencia comunicación lingüística a través del cuaderno del alumno y del cuento motor en el área de EF.

\section{¿QUÉ SON LAS COMPETENCIAS BÁSICAS?}

La Ley Orgánica de Educación (LOE 2/2006, de 3 de mayo), sitúa las competencias básicas (a partir de ahora, CC.BB.) como un elemento curricular de importancia capital. Así, el artículo $6^{\circ}$, define de forma explícita el currículo como «El conjunto de objetivos, competencias básicas, contenidos, métodos pedagógicos y criterios de evaluación». El currículo deberá incluir, de acuerdo con lo recogido en el Anexo I del Real Decreto 1513/06, de 7 de Diciembre, por el que se establecen las enseñanzas mínimas en la Educación Primaria, al menos las siguientes competencias básicas:

1. Comunicación lingüística.

2. Matemática.

3. Conocimiento e interacción con el mundo físico.

4. Tratamiento de la información y competencia digital.

5. Social y ciudadana.

6. Cultural y artística.

7. Aprender a aprender.

8. Autonomía e iniciativa personal. 
En dicho anexo se definen las competencias como "aquellos aprendizajes que se consideran imprescindibles, desde un planteamiento integrador y orientado a la aplicación de los saberes adquiridos. Éstas son las que debe haber desarrollado un joven o una joven al finalizar la enseñanza obligatoria para poder lograr su realización personal, ejercer la ciudadanía activa, incorporarse a la vida adulta de manera satisfactoria y ser capaz de desarrollar un aprendizaje permanente a lo largo de la vida".

Morocho Ponce, Miguel (2010), en Lecturas: Educación Física y Deportes, Revista Digital, 140. cita que la Comisión Europea en "Eficiencia y equidad en los sistemas europeos de educación y formación" (2006), define la competencia clave o básica, como una "combinación de destrezas, conocimientos y actitudes adecuadas al contexto". Completa la definición dada anteriormente por el RD de enseñanzas mínimas, diciendo que las competencias clave "...son aquellas que todas las personas precisan... para la inclusión social y el empleo. Éstas deberían haber sido desarrolladas para el final de la enseñanza o formación obligatoria en la medida necesaria para la vida adulta y deberían seguir desarrollándose, manteniéndose y actualizándose, como parte de un aprendizaje a lo largo de la vida".

La Organización para la Cooperación y el Desarrollo Económico (OCDE) elaboró el proyecto DESECO (Definición y selección de competencias, 2002) y nos dice que "ser competente" es "ser capaz de responder a demandas complejas y llevar a cabo tareas diversas de forma adecuada".

Ruiz Nebrera, J.J. (2008), en Lecturas: Educación Física y Deportes, Revista Digital, 127, nos dice que DESECO define las competencias básicas como "conjunto complejo de conocimientos, habilidades, actitudes, valores, emociones y motivaciones que cada individuo o cada grupo pone en acción en un contexto concreto para hacer frente a las demandas peculiares de cada situación. Así, se consideran competencias fundamentales aquellas competencias imprescindibles que necesitan todos los seres humanos para hacer frente a las exigencias de los diferentes contextos de su vida como ciudadanos". Las competencias fundamentales o "key competencias" son aquellas que son importantes para muchas áreas de la vida, que contribuyen a una vida satisfactoria y al buen funcionamiento de la comunidad social.

También deben contribuir a transformar el concepto tradicional de enseñanza, basado en la adquisición de conocimientos, en un concepto moderno de aprendizaje, basado en la capacidad de resolver situaciones a lo largo de la vida.

Las competencias básicas se sustentan en la realización personal, la inclusión social y la ciudadanía activa, y contribuyen a adaptar el proceso de enseñanzaaprendizaje a la sociedad actual.

\section{¿QUÉ CARACTERÍSTICAS Y FINALIDADES TIENEN LAS COMPETENCIAS BÁSICAS?}

Como características comunes a todas ellas podemos destacar:

- Proporcionan la capacidad de saber hacer, es decir, de aplicar los conocimientos a los problemas de la vida profesional y personal. Incluyen una combinación de saber, habilidades y actitudes.

- Pueden ser adquiridas en todo tipo de contextos: escuela, hogar y ámbitos extraescolares. 
- Son multifuncionales, ya que pueden ser utilizadas para conseguir múltiples objetivos.

- Tienen un carácter integrador, aunando los conocimientos, los procedimientos y las actitudes (saber, ser, saber hacer).

- Permiten integrar y relacionar los aprendizajes con distintos tipos de contenidos, utilizarlos de manera efectiva y aplicarlos en diferentes situaciones y contextos (aplicabilidad y transferencia).

- Se deben aprender, renovar y mantener a lo largo de toda la vida.

- Constituyen la base de los aprendizajes básicos posteriores.

- Se inspiran en la teoría relacionada con el aprendizaje basado en competencias (Competency Based Training).

- En definitiva, pretenden que se adquieran e integren las tres formas contemporáneas del saber:

- Saber teórico (conocimientos). SABER.

- Saber práctico (habilidades y destrezas). SABER HACER O SABER COMO HACER.

- Saber ser (actitudes). SER O QUERER SER.

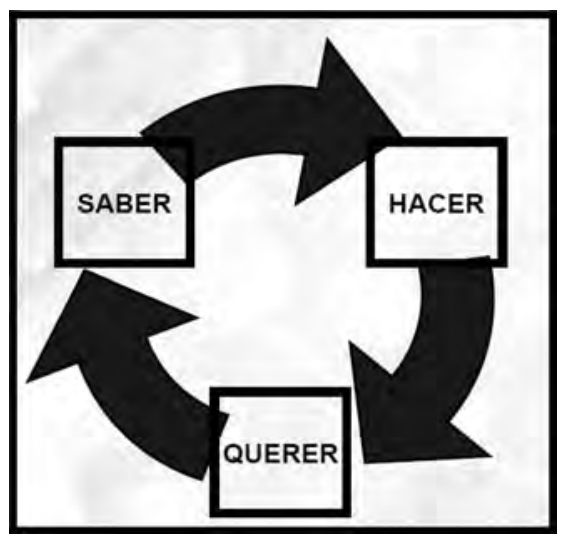

Imagen 1. Formas contemporáneas del saber.

La inclusión de las competencias básicas en el currículo tiene varias finalidades fundamentales:

- Integrar los diferentes aprendizajes, tanto los formales -incorporados a las diferentes áreas- como los informales y no formales.

- Permitir a todos los alumnos y alumnas integrar sus aprendizajes, ponerlos en relación con distintos tipos de contenidos y utilizarlos de manera efectiva cuando les resulten necesarios en diferentes situaciones y contextos.

- Orientar la enseñanza al permitir identificar los contenidos y los criterios de evaluación que tienen carácter imprescindible y, en general, inspirar las distintas decisiones relativas al proceso de enseñanza y aprendizaje. 


\section{¿QUÉ ES LA COMPETENCIA COMUNICATIVA Y LINGÜÍ́STICA?}

Hymes (1989), define la competencia comunicativa como "la capacidad de elaborar, producir y descodificar mensajes, discursos adecuados, tanto en el ámbito de la oralidad como de la escritura". Mensajes que cumplan con la eficacia comunicativa, en el sentido de interacción con las demás personas de su entorno.

La competencia comunicativa supone poder manejar distintos niveles del código según las situaciones comunicativas en las que deba desenvolverse el hablante. Por tanto, la competencia comunicativa presupone las siguientes subcompetencias: las denominadas lingüísticas o gramaticales (que se refiere al conocimiento del sistema de la lengua), sociolingüística (que proporciona mecanismos de adecuación a la situación y el contexto), discursiva (que rige la coherencia y cohesión de los diversos tipos de discurso) y estratégica (que regula la interacción y permite reparar o contrarrestar las dificultades o rupturas en la comunicación).

\section{Competencia en la comunicación lingüística en el Real Decreto 1513/2006 (anexo II, áreas curriculares)}

En la introducción del RD 1513/2006, dedicada a la materia de Educación Física, se cita textualmente: "El área también contribuye, como el resto de los aprendizajes, a la adquisición de la competencia en comunicación lingüística, ofreciendo gran variedad de intercambios comunicativos, del uso de las normas que los rigen y del vocabulario específico que el área aporta".

Citando a Ruiz Nebrera, J.J. (2008), en Lecturas: Educación Física y Deportes, Revista Digital, 127, dicha competencia básica se refiere a:

- Utilización del lenguaje como instrumento de:

- Comunicación oral y escrita.

- Representación, interpretación y comprensión de la realidad.

- Construcción y comunicación del conocimiento.

- Organización y autorregulación del pensamiento, de las emociones y de la conducta.

- Comprensión y expresión lingüística de los contenidos y aplicación a distintos contextos de la vida.

- Hábitos de lectura y escritura.

- Actitudes de escucha, exposición y diálogo, teniendo en cuenta y respetando las opiniones de los demás.

- Dominio de la lengua oral y escritura en múltiples contextos.

- Uso funcional de, al menos, una lengua extranjera.

\section{DesarRollo de la COMPETENCIA de la COMUNICACIÓN liNGÜÍSTICA A TRAVÉS DE LA EXPRESIÓN CORPORAL}

Si analizamos los elementos básicos del currículum del RD 1513/2006 destinado al área de EF, comprobamos que en el primer ciclo hay referencias explícitas al 
desarrollo de la competencia básica "comunicación lingüística". Concretamente, en el bloque de contenidos 3: Actividades físicas artístico-expresivas, encontramos contenidos como:

- Descubrimiento y exploración de las posibilidades expresivas del cuerpo y del movimiento.

- Exteriorización de emociones y sentimientos a través del cuerpo, el gesto y el movimiento, con desinhibición.

- Imitación de personajes, objetos y situaciones.

- Posibilidades expresivas con objetos y materiales.

Asimismo, se descubre un criterio de evaluación ( $n^{\circ}$ 7) en primer ciclo, que guarda una relación directa con la competencia básica aquí tratada:

- Simbolizar personajes y situaciones mediante el cuerpo y el movimiento con desinhibición y soltura en la actividad.

Es importante que sean capaces de experimentar con el propio cuerpo y tomar conciencia de sus posibilidades expresivas a través del gesto y el movimiento. Se valorará la aportación espontánea y el esfuerzo para encontrar nuevas formas expresivas que se alejen de situaciones estereotipadas. Asimismo, se tendrá en cuenta el esfuerzo personal para implicarse en las propuestas y sentirse "dentro del personaje", aceptando el papel que toque representar sin prejuicios de ninguna índole.

\section{¿QUÉ ES UN CUENTO MOTOR?}

Según Puertas Sevilla, Ma Carmen (2009), en la Revista Digital "Sapientia", el cuento motor "es un cuento que, al ser narrado con ayuda de música favorece diversidad de movimientos, dramatizaciones y simulaciones haciendo protagonista a los propios niños".

Son una herramienta pedagógica para que nuestros alumnos/as exploren sus posibilidades motrices y creativas, para favorecer su autonomía, y permite globalizar la enseñanza partiendo del centro de interés, en este caso el cuento, relacionando los contenidos de distintas áreas de la Educación Primaria.

Según Conde (2001), un cuento motor podríamos definirlo como "un cuento jugado, un cuento vivenciado de manera colectiva, con unas características y unos objetivos propios".

Entre los objetivos que pretenden desarrollar los cuentos motores están, según Conde (2001):

- Desarrollar los elementos psicomotores básicos (esquema corporal, lateralidad, estructuración espacial y temporal y ritmo), las cualidades motrices coordinativas (coordinación y equilibrio), las habilidades básicas (desplazamientos, saltos, lanzamientos, recepciones y giros), las habilidades genéricas (bote, conducciones, golpeos, etc.).

- Desarrollar las capacidades físicas básicas de manera indirecta.

- Sentar las bases preventivas e higiénicas de la salud a través del ejercicio físico desde las primeras edades y como un hábito de vida. 
- Desarrollar la capacidad creativa del niño/a, haciéndole interpretar corporalmente lo que se le está verbalizando, potenciando el desarrollo de su imaginación y construyendo sus capacidades cognitivas.

- Globalizar la enseñanza de las áreas musical, plástica y dramatización y lengua, entre otras, partiendo del centro de interés: el cuento infantil.

- Descubrir el propio cuerpo como vehículo de comunicación.

- Enfrentar al alumno/a a la toma de sus propias decisiones en cuanto a expresión y movimiento, tomando conciencia de su cuerpo y del espacio que le rodea.

- Interdisciplinar otras áreas, principalmente Música y Plástica, con el fin de globalizar la enseñanza.

Deberemos de tener en cuenta algunos requisitos necesarios a la hora de transmitir el cuento, entre los que enumera Martínez (2007):

- La persona responsable de la actividad ha de conocer muy bien el cuento, con el fin de mantener una dinámica flexible pero sin perder de vista los objetivos.

- Disponer a priori del material que se vaya a utilizar y preparar su ubicación y utilidad.

- Disponer de un espacio amplio y/o abierto.

- Integrarse como uno más en la práctica de la actividad.

- La sesión deberá seguir una estructura dividida en tres partes (fase de animación, fase principal o de desarrollo de la actividad, y vuelta a la calma).

- Una vez finalizada la sesión, podemos mantener una charla con los niños/ as (dispuestos en corro) y trabajar así otros contenidos más relacionados con la compresión y expresión orales.

- Esta actividad es generadora de otras muchas que podemos aprovechar según intereses y circunstancias.

\section{El CUENTO MOTOR Y EL CUADERNO DEL ALUMNO COMO MEDIO DE DESARROLLO DE LA COMPETENCIA DE LA COMUNICACIÓN LINGÜÍSTICA}

A continuación se muestra un ejemplo del cuaderno del alumno correspondiente a la unidad didáctica "Explosión de expresiones", perteneciente al bloque de contenidos de expresión corporal, destinado al $2^{\circ}$ nivel de Educación Primaria.

Este cuadernillo se imprime, por economía, en blanco y negro, coloreando los alumnos la portada. La impresión se realiza con dos hojas en una para que el tamaño del cuadernillo resulte de medio folio, grapando las hojas en el centro. Al final de curso el maestro de Educación Física, en colaboración con el tutor, encuadernarán todos los cuadernillos de todas las unidades didácticas, quedando como resultado un cuaderno final del área de EF, cuya portada será de realización libre por cada alumno.

En esta unidad didáctica, "Explosión de expresiones", el trabajo del cuadernillo será posterior al trabajo en la clase de EF para reforzar y evaluar también los contenidos pretendidos en esta área. 
Se trabaja en estrecha colaboración y coordinación con el tutor del grupo, a través de un trabajo interdisciplinar con el resto de áreas (lenguaje, conocimiento del medio, matemáticas y expresión artística), ya que en las reuniones de ciclo se repasan los contenidos a trabajar y reforzar, y nosotros, a partir de las sesiones de EF y con la elaboración del cuadernillo por parte del alumno, buscamos el desarrollo de los mismos. El tutor trabajará la lectura del cuento del cuadernillo, que en el caso que nos ocupa se trata de un cuento motor, que los niños han realizado anteriormente en la clase de EF. Se trabajará con ellos la lectura, la entonación, así como la comprensión lectora, y se les explicará las actividades a realizar en relación con los contenidos del área de lengua, matemáticas, artística y conocimiento del medio.

Los alumnos terminarán la realización de las actividades en casa y las entregarán al maestro tutor, que posteriormente se las dará al maestro de EF para su corrección. Estos dos maestros reflexionarán sobre los resultados en la posterior reunión de ciclo.

El maestro de EF tiene una lista de control donde refleja la entrega y realización de tareas competenciales de los cuadernillos, así como un criterio de evaluación en su programación donde se refleja la adecuada realización de las mismas. Se requiere la máxima colaboración con el tutor del grupo, ya que pretendemos una enseñanza global, contextualizada y significativa, y pretendemos desde el área de la EF contribuir al máximo al desarrollo de las competencias básicas sin restar tiempo a la actividad motriz. 


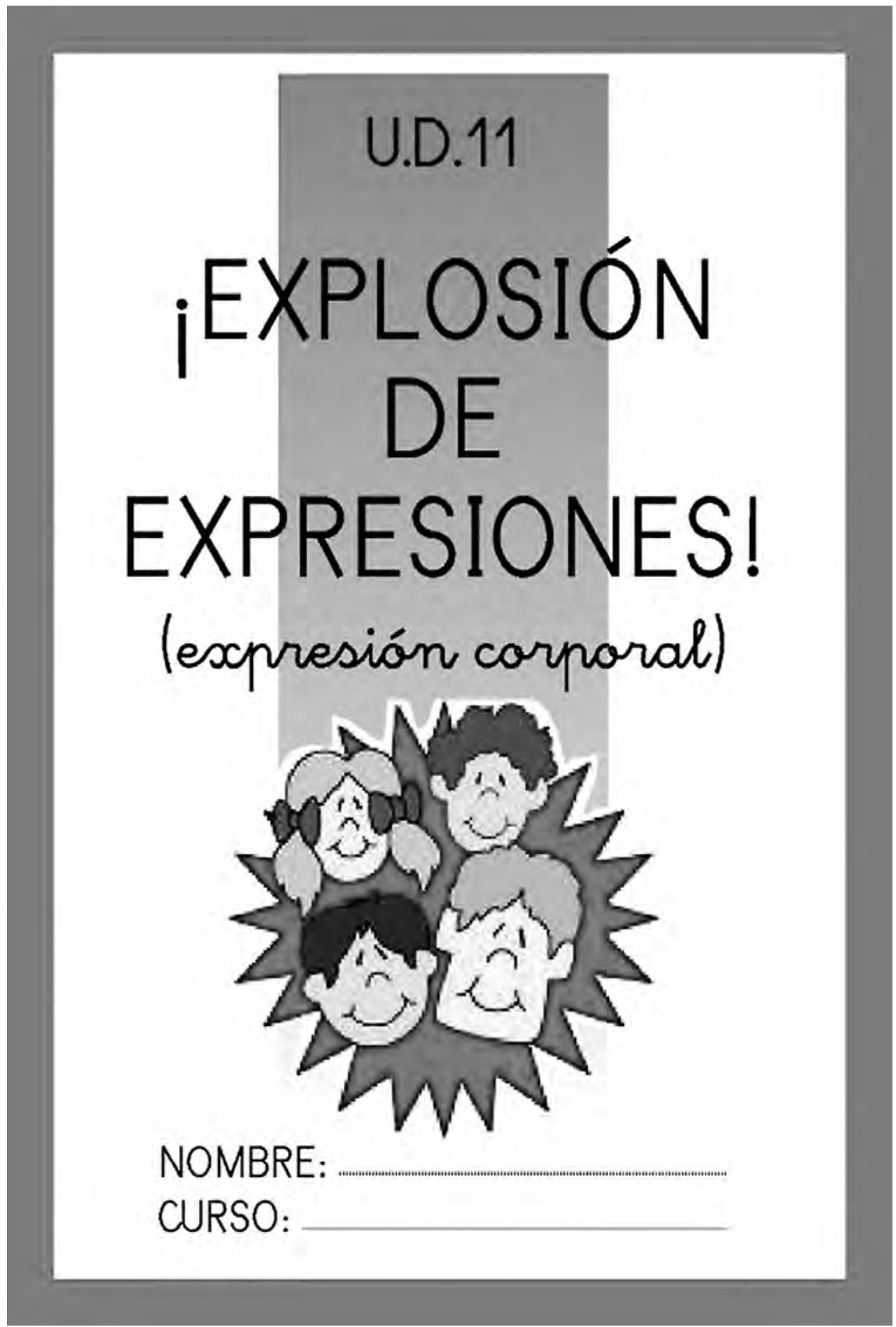

Imagen 2. 
EVA RIVAS MARTÍNEZ - JAVIER TERROBA VALDEMOROS

¿QUÉ PASA AQUII?

Un día desperté y me encantré can ésto:

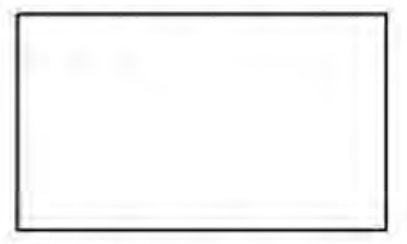

Pera... ¿qué pasa?, me pregunté:

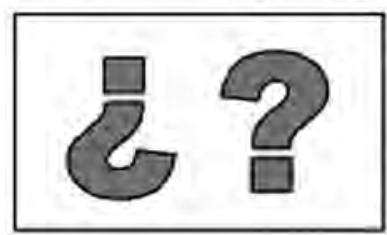

¿Creéis que era de nache?

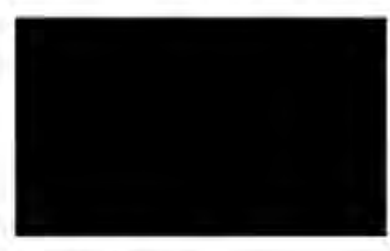

¿Se había ida la ?

¿Se había apagada el ?

Yo no entendía nada, asi que se me quedá esta cara:

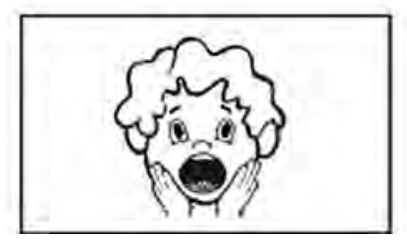

Imagen 3.

Contextos Educ., 13 (2010), 155-174

164 
DESARROLLO DE LA COMPETENCIA BÁSICA “COMUNICACIÓN LINGÜÍSTICA” A TRAVÉS DEL CUENTO MOTOR...

Después del susta, mi cara fue de preacupación:

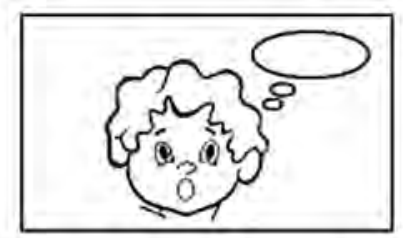

y más tarde mi cara fue de tristera:

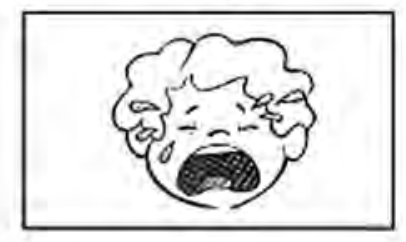

Alga tenía que haces, no se la que habia pasada, pera de repente estaba yo sala y no había nada, ni nadie.

Me puse a caminar y me di cuenta, que sóla padía pisar unas que habia en el suela (jauidada chicas!, qué suerte tenéis que estáis en ese círcula encima de las ACTIVIDAD:

Paca a paca tada el suela se cubrió cama de una manta muy suave que daba gusta tacarla.

Imagen 4.

165

Contextos Educ., 13 (2010), 155-174 


\section{ACTIVIDAD:}

Me he divertida un vato, pera aquí sigue sin haber nada, una atra Pues can estas y lac de antes alga padré hacer...

\section{ACTIVIDAD:}

Yo he visto unas gardas, que yo crea quesan II]. ¿qué as parece si la plantamas y camienza a tener alga en este blanca? Vamas a plantar cuatro, una en cada esquina, a cada grupa as vay a dar un I una Tadas tenéis que calabarar. ACTIVIDAD:

(música de las 7 enanitas).

Pera cuidada, parque pranta llegué a un sitia dande había pinchas en el suela.

\section{Imagen 5.}




\section{ACTIVIDAD:}

Las pinchas desaparecieran pera pranto crecieran unas - altas, casi na me dejaban ni andar, ni ver, jeuidada na as chaquéis!. ACTIVIDAD:

Ohhhhll, se me hunden las piernas..., iqué pasa chicas?

ACTIVIDAD:

Cada vez hay más barra, cada vez está más líquida... Llegamas a un ¡Qué divertida!, pademas jugar en el agua. ACTIVIDAD:

¿Qué pademas hacer en el agua? ACTIVIDAD:

Está empezanda a saplar el iqué les pasa a los It? 
EVA RIVAS MARTÍNEZ - JAVIER TERROBA VALDEMOROS

ACTIVIDAD:

Y si vamas andanda par la calle y empieza a saplar tan fuerte el éc y más y más... ACTIVIDAD:

Se me ha abvidada preguntaras antes... Ya he plantada un manzana.

Se me acurre que pademas coger del is una fruta, ¿cáma?

ACTIVIDAD:

Ya hemas merendada, pera... ¿qué vea?, una

- ¿Cóma pademas subir?

ACTIVIDAD:

Música de Misión Impasible.

Está empezanda a

La

ha quedada campletamente nevada.

¡A jugar!

Imagen 7.

Contextos Educ., 13 (2010), 155-174

168 


\section{ACTIVIDAD:}

¿Qué departe se practica en la nieve?

Y para en la $/ 1 /$ más alta, jcáma subimas?

\section{ACTIVIDAD:}

¿Sabéis la que me ha pasada?, que al caer del telesillas ya la vea toda... Las espalderas, las (1) ,... ¿Sabéis la que me había pasada?

¡Qué me había metida en un libra en blanco! 
EVA RIVAS MARTÍNEZ - JAVIER TERROBA VALDEMOROS

ACTIVIDADES

¿Qué pasá cuanda me desperté?

¿Cuál fue mi primera cara?

¿Par qué crees que me preacupé y me puse triste?

¿Qué casas se pueden hacer can unas tablas y unas cuerdas?

¿Cóma se plantaran las árboles?

¿Qué departe se practica en una mantaña nevada?

¿En qué estación del año?

¿Cóma carreré más, esquianda en línea recta a en curvas?

Imagen 9.

Contextos Educ., 13 (2010), 155-174

170 
DESARROLLO DE LA COMPETENCIA BÁSICA “COMUNICACIÓN LINGÜÍSTICA” A TRAVÉS DEL CUENTO MOTOR...

¿Qué casas pueda simbalizar can la mana?

Escribe sentimientas que puedes expresar can la cara.

¿Qué puede expresar un abraza?

¿Cáma se llaman las palabras que expresan más de un significado?

Par ejemplo: BANCO. Pon tú atra ejemplo.

Pan el artícula a cada nambre; EL, LA, LOS, LAS:

emación

cariño

abrazas

cancianes besas

amar

amistad amigas

Imagen 10.

171

Contextos Educ., 13 (2010), 155-174 
EVA RIVAS MARTÍNEZ - JAVIER TERROBA VALDEMOROS

Escribe una palabra detrás de cada artícula que tenga que ver con las emaciones.

$$
\text { UN }
$$

UNOS

UNA

UNAS

Escribe una palabra can cada silaba:

GE:

GI:

JE:

JI;

Si me das tres besas y ya te day el dable, ¿cuántos te daré?

Si te day cuatra abrazas y tú me das la mitad, ¿cuántas me darás?

Tenga cuatro amigas, tú tienes el triple. ¿Cuántas tienes?

Imagen 11.

Contextos Educ., 13 (2010), 155-174

172 


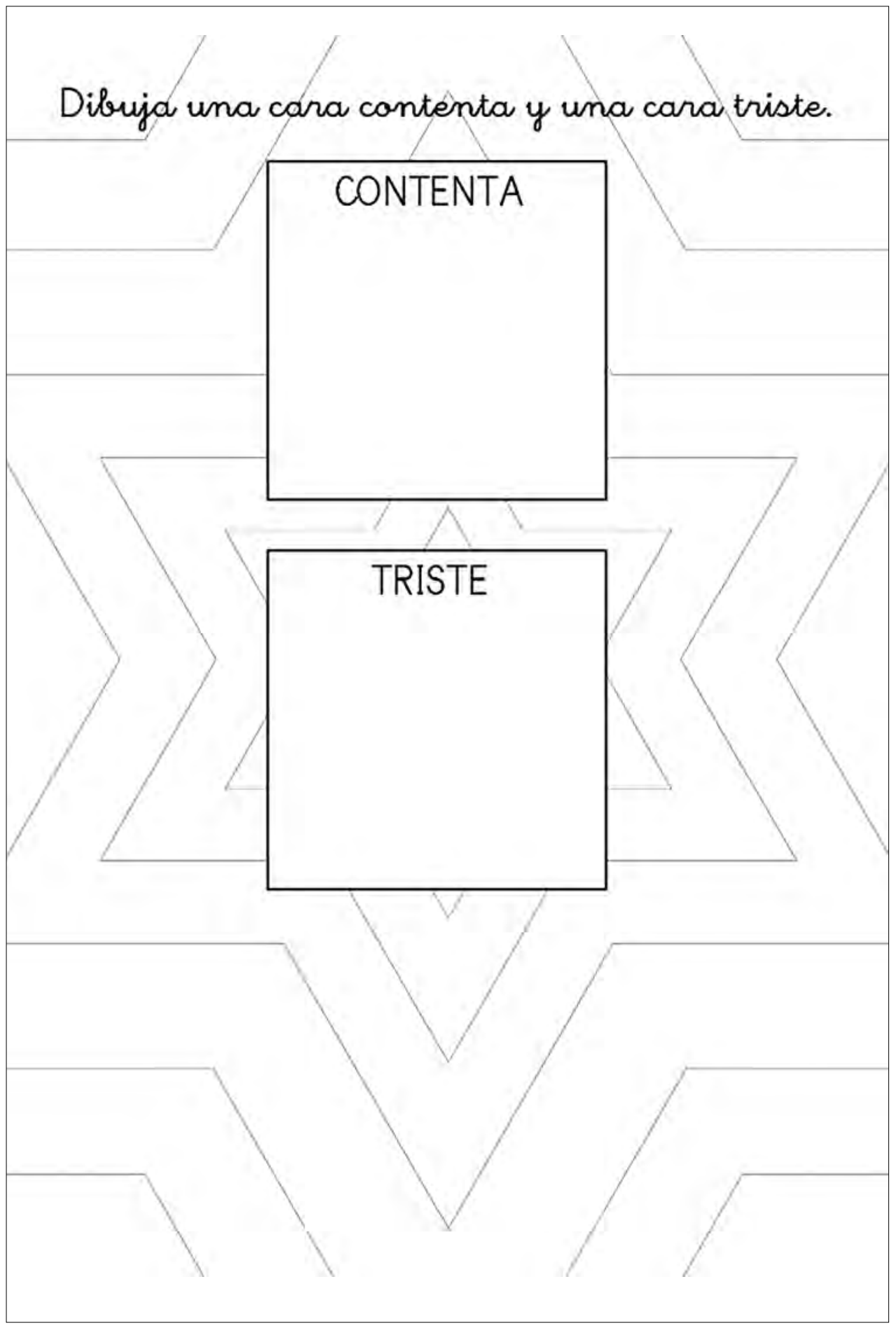

Imagen 12. 


\section{REFERENCIAS BIBLIOGRÁFICAS}

CÓNDE, J. L. (2001). Cuentos motores. Vol. I y II. Barcelona: Paidotribo.

HYMES, D. (1989). Postcript. Applied Linguistics, 2, 244-250.

LEY ORGÁNICA 2/2006, DE 3 DE MAYO, DE EDUCACIÓN (B.O.E. 4 de Mayo de 2006).

MARTíNEZ CALLE, A. (2007). Cuentos motores. Sevilla: Wanceulen.

MOROCHO, M. (2010). Las competencias básicas en Educación Secundaria en la materia de Educación Física en la Comunidad Autónoma de Andalucía. Lecturas: Educación Física y Deportes, Revista Digital, 140 [Disponible en: http://www.efdeportes.com].

ORGANIZACIÓN PARA LA COOPERACIÓN Y EL DESARROLLO ECONÓMICO (2002). Definición y selección de competencias. Proyecto DESECO.

PUERTAS, M. C. (2009). El cuento motor. Revista Digital "Sapientia" [Disponible en: htp://www.feteugtalmeria.org/revistadigital].

REAL DECRETO 1513/2006, DE 7 DE DICIEMBRE, por el que se establecen las enseñanzas mínimas de la Educación Primaria. (B.O.E., 8 de diciembre de 2006).

RUIZ NEBRERA, J. J. (2008). Las competencias básicas en la educación primaria. Lecturas: Educación Física y Deportes, Revista Digital, 127. [Disponible en: http://www.efdeportes.com]. 